Noname manuscript No.

(will be inserted by the editor)

\title{
A Novel Micromechanical Model of Nonlinear Compression Hysteresis in Compliant Interfaces of Multibody Systems
}

\author{
Ramin Masoudi • John McPhee
}

Received: date / Accepted: date

\begin{abstract}
A micromechanical model of nonlinear hysteretic compression between interacting bodies of multibody systems, covered with fibrous structures, has been created and validated experimentally in this work. As an application, a multibody dynamic model of an upright piano action mechanism with felt-covered contacting bodies is considered, and the obtained results were verified using experiments. Felt, as a typical nonwoven fiber assembly, has been used in various contact surfaces of piano action mechanisms to transfer the force applied on the key to other components, smoothly and continuously. To keep the simulation time tractable in the mechanistic multibody dynamic model, interaction between felt-lined interfaces has to be simplified enough so that in each step of simulation time, contact forces can be calculated as a function of penetration depth between colliding objects. The developed micromechanical approach is capable of estimating nonlinear bulk response of felt in terms of microstructural parameters of the network, assuming a binomial distribution of the number of fiber contacts and bending of constituent fibers. Hysteresis is included based on a fiber-to-fiber friction approach, which generates a speed-independent response to compressive loading schemes, as has been observed in experiments. A computational algorithm is introduced to apply the sophisticated hysteretic micromechanical model to the multibody systems simulation, including transitions between loading-unloading stages.
\end{abstract}

Keywords Micromechanics of contact - Multibody systems · Compression hysteresis, Felt models

R. Masoudi

200 University Avenue West, Waterloo, ON, Canada

Tel.: +1 (519) 888-4567 ext. 37099

Fax: +1 (519) 746-4791

E-mail: rmasoudi@uwaterloo.ca

J. McPhee

200 University Avenue West, Waterloo, ON, Canada

Tel.: +1 (519) 888-4567 ext. 35341

Fax: +1(519) 746-4791

E-mail: mcphee@uwaterloo.ca 


\section{Introduction}

Contact dynamics has been a challenging issue in the dynamic analysis of multibody systems, with a broad application in robotics, vehicle and train dynamics, biomechanics, and aerospace engineering. Mathematically, contact can be introduced as unilateral constraints in multibody dynamics, i.e. the topology of the system will vary during the simulation. There are several modeling schemes to include contact phenomena in the physics-based modeling of multibody systems, of which the regularized approach, also called continuous analysis, has shown promising performance in terms of modeling accuracy and computational efficiency. Simplistic equivalent force-deformation systems including the hysteresis effect (e.g. nonlinear spring-damper models), which relate the dynamic contact force to the relative penetration depth and rate of contacting bodies $[1,2]$, are among the well-known modeling schemes for this force-based approach. In the regularized method, continuous force-deformation relations govern interaction evolution between bodies based on the compliance and damping properties of contact interfaces. Hysteresis generated by the models presented in Refs. [1,2] is speed-dependent, implying viscoelastic behavior of the materials in contact.

The contact problem is more complicated when the energy loss observed in the loading-unloading process is speed-independent. In this case, the source of hysteresis can be attributed to dry friction between micro-structure components of interfaces. Felt, a nonwoven fibrous material formed by interlocked wool fibers, possesses such mechanical properties in compressive loading schemes. As shown in Fig. 1, our experiments using Scanning Electron Microscopy (SEM) illustrates the micro-structure of a typical felt sample, which is used in lining piano hammers [3]. Based on a superficial analysis from the images, the fiber surface seems to be rough enough to cause considerable friction between interacting fibers.
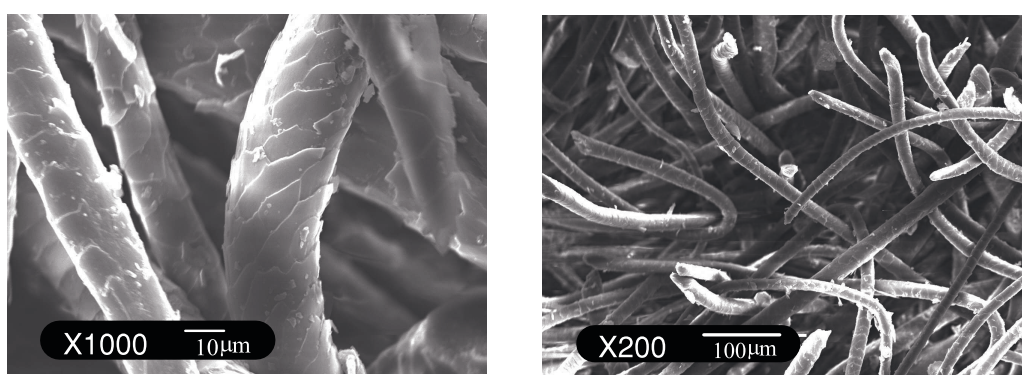

Fig. 1 Scanning Electron Microscopy (SEM) of a typical gold-coated felt sample.

At the macro-scale (as shown in Fig. 2), an impact test with a piano feltcovered hammer striking a rigid stop reveals the existence of nonlinear hysteresis independent of speed of deformation, which affirms the hypothesis from the microlevel observations. It means that a viscoelastic relation to include hysteresis is not suitable for felt compression analysis.

In this study, the main objective is to develop a micromechanical model of felt compression, applicable to contact dynamics of multibody systems. Model- 


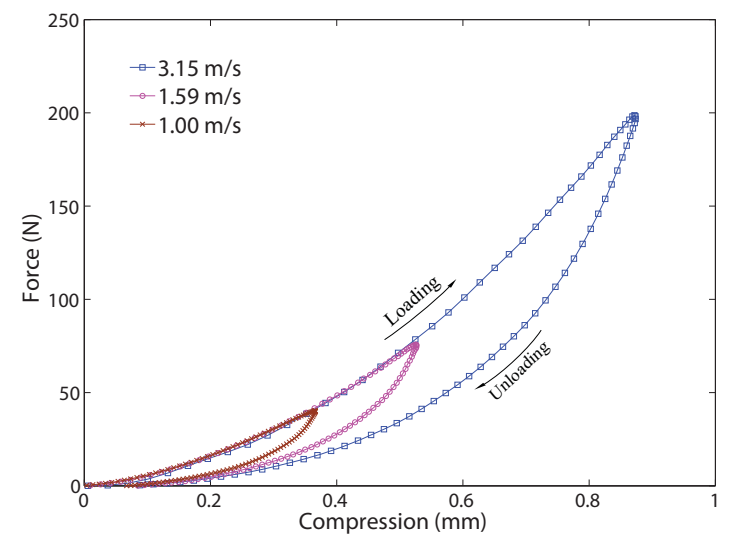

Fig. 2 Loading and unloading curves for dynamic force-compression of a piano hammer impacting a rigid stop at various speeds.

ing felt compression versus force characteristics is a complex and computationally expensive process, so simplification scenarios such as statistical and probabilistic techniques are incorporated to derive a force-compression relation that can be updated at every step of the multibody simulation in a sensible pace. To include hysteresis, we will focus on the friction between interacting fibers, assumed to be of the Coulomb type in which the frictional force is proportional to the applied normal force, independent of the contact area. Furthermore, nonlinear compliance observed in both loading and unloading stages is modeled mechanistically to provide a fully physics-based contact model.

To establish the validity of the micromechanical model, an upright piano action is considered. A piano action mechanism transmits the force applied on the key to a felt-covered hammer that strikes the strings to produce a desired sound. The interconnected bodies of the action must satisfy geometric and dynamic requirements in order to transfer the expected motions and generate the desired response. To allow a softer feel and reduce mechanical noise in the action, motion transition between different bodies is formed through contact surfaces covered with felt or similar fiber assemblies. Consequently, an accurate simulation model must involve a mechanistic contact approach to capture intermittent loading-unloading during the bodies' interaction. Estimating the value of contact forces in terms of the deformation of felt between colliding bodies in the dynamic simulation of the action motivates a systematic investigation into the nonlinear behavior of these contact interfaces. To be mechanistic, the developed contact model employs microstructural features in the response of the network, and on the other hand, it should be simple enough to estimate the contact forces using computationally low-cost algorithms in multibody dynamic simulations.

\section{Micromechanics of random fiber networks including hysteresis}

There have been various challenging issues in micromechanical modeling of nonwoven fiber assemblies: highly nonlinear compliance, nonlinear hysteretic phenomena, 
randomness of the network structure, complex morphologies, and dominant microstructural deformation schemes. In micromechanical approaches, response of the fiber mass to external loads at the macro-level is evaluated based on the reaction of its constitutive fibers at the micro-level. To investigate macrostructural behavior of fiber assemblies properly and precisely, one has to characterize the network microscopically, including fiber distribution and interaction in a typical control volume of the continuum media, as well as mechanical and geometrical characteristics of each individual fiber.

Felt, as a typical fiber mass, is defined by Lehmberg [4] as "a fibrous material built up of interlocked wool fibers by mechanical and chemical action, moisture and heat". Due to its excellent damping properties, felt has been used widely in contact between interacting bodies of mechanical systems. Musical instruments, particularly piano mechanisms, use felt-covered contact interfaces in suppressing mechanical impact, vibrations, and most tangibly, undesired noises from component wear and tear. There are numerous mechanistic models developed based on response of the fibers at the micro-level, but none of them are suitable for studying macro-level compression behavior of felt. Stamm [5] showed that the micromechanical approaches developed by van Wyk [6], Neckáŕ et al. [7], and Komori et al. $[8,9]$ failed to match experimental results for felt compression. Furthermore, Masoudi [3] utilized a more sophisticated model proposed by Alkhagen et al. [10], which was based on a constitutive relation for a fiber mass using the rate theory of flexible granular solids, but unsatisfactory results were obtained for compression of felt with various volume fractions. This inconsistency between the experimental results and micromechanical models can be attributed to ill-defined schemes in characterizing the structure of the random fiber network, particularly in estimating the number of fiber-to-fiber contacts for felt materials.

In this paper, the general focus is on felt as a statistically homogenous fiber network in which the fibers disperse in the sense that there is no correlation between the spatial and orientation distributions. There can be considered as well a length scale on which the fibers can be considered straight. Accordingly, there is a mean distance between contact points due to interaction between these fiber segments, called mean free fiber length, which has a pivotal role in constructing the micromechanical model. Moreover, we assume that the fiber diameters are constant (considering an average value for the experimentally measured fiber diameters) and their mechanical properties do not change during compression [3].

\subsection{Number of fiber contacts - mean free fiber length}

A crucial step in micromechanical analysis of fiber assemblies is to construct a network based on statistical and geometrical properties of the fiber mass. Estimating the number of fiber contacts, which directly affects mean free fiber length, plays a key role in estimating microscopic response of the network in terms of microstructural features of constituent fibers.

Binomial distribution of the number of contact points on a particular fiber is a promising idea that leads to a complicated, but more accurate model compared to existing models, particularly in case of the dense fiber networks [11]. In general, the random distribution of the number of contacts on any arbitrary fiber with direction 
$(\theta, \phi)$ (shown in Fig. 4) can be expressed according to the Poisson distribution:

$$
\hat{P}(k ; \vartheta)=e^{-\vartheta} \frac{\vartheta^{k}}{k !}, \quad \vartheta=\frac{8 v_{f} \delta_{f} J(\theta, \phi)}{\pi d_{f}}
$$

with the mean value of $\vartheta$ and number of exact occurrences $k$, the probability of which is given by the function $\hat{P}, v_{f}$ is the volume fraction defined as the volume of the fiber per network volume, $J(\theta, \phi)=\int_{0}^{\pi} \int_{0}^{\pi} \sin \hat{\kappa}_{\left(\theta, \phi ; \theta^{\prime}, \phi^{\prime}\right)} \Omega\left(\theta^{\prime}, \phi^{\prime}\right) \sin \theta^{\prime} \mathrm{d} \theta^{\prime} \mathrm{d} \phi^{\prime}$ is a structural parameter, $\hat{\kappa}_{\left(\theta, \phi ; \theta^{\prime}, \phi^{\prime}\right)}$ is the angle between two spatial fibers with orientations $(\theta, \phi)$ and $\left(\theta^{\prime}, \phi^{\prime}\right), \Omega(\theta, \phi)$ is the orientation distribution function, $\delta_{f}$ is the length of the straight fiber segment, and $d_{f}$ is the fiber diameter. In this statistical approach, there are no constraints imposed by the fibers that are already in contact with the arbitrary fiber. So, a modification was introduced by Neckár et al. [11] to achieve a more realistic model, based on a binomial distribution of the number of contact points on the fiber. Accordingly, the distribution of the number of contact points on the fiber can be estimated by the binomial distribution

$$
B\left(k ; v_{m}, S(\vartheta)\right)=\left(\begin{array}{c}
v_{m} \\
k
\end{array}\right) S(\vartheta)^{k}(1-S(\vartheta))^{v_{m}-k}, \quad S(\vartheta)=1-e^{-\frac{\vartheta}{v_{m}}}
$$

with the mean value of $\bar{m}=S(\vartheta) v_{m}=v_{m}\left(1-e^{-\frac{\vartheta}{v_{m}}}\right)$, in which $v_{m}$ is the maximum number of contact points that could be created on a fiber length $\delta_{f}$.

Applying statistical and mathematical approaches, the number of contacts per unit volume, $N_{v}$, and mean free fiber length, $\bar{\lambda}$, can be acquired as [11]:

$$
N_{v}=\frac{2 v_{f}\left(1-e^{-2 v_{f} \gamma}\right)}{\pi d_{f}^{3} \gamma} \quad \Rightarrow \quad \bar{\lambda}=\frac{\gamma d_{f}}{\left(1-e^{-2 v_{f} \gamma}\right)}
$$

where $\gamma=\delta_{f}\left(v_{m} d_{f}\right)^{-1}$.

\subsection{Theoretical deformation theory based on fiber bending}

The relation between macroscopic pressure and bulk deformation in fiber networks was originally developed by van Wyk, based on fiber bending as the dominant microscale deformation scheme [6]. The general idea is to consider fiber segments as Euler-Bernoulli beam elements supported at numerous points of contact with other fibers, in which the segments could be straight or curved and boundary conditions at contact points could be estimated as simply-supported, free, or built-in ends.

To develop a mechanistic model, a horizontal beam supported at contact points equally spaced at distance $2 \bar{\lambda}$ apart is considered, along with built-in ends at non-sliding contact points. A transverse contact force $F_{Y}$ acting at the midspan of the beam results in a deformation $\delta_{Y}$ according to beam formulas, based on infinitesimal strain theory, as

$$
F_{Y}=\frac{192 E I}{(2 \bar{\lambda})^{3}} \delta_{Y}
$$

where $E$ is the modulus of elasticity of the fiber and $I$ is the second moment of area of the fiber cross section. 


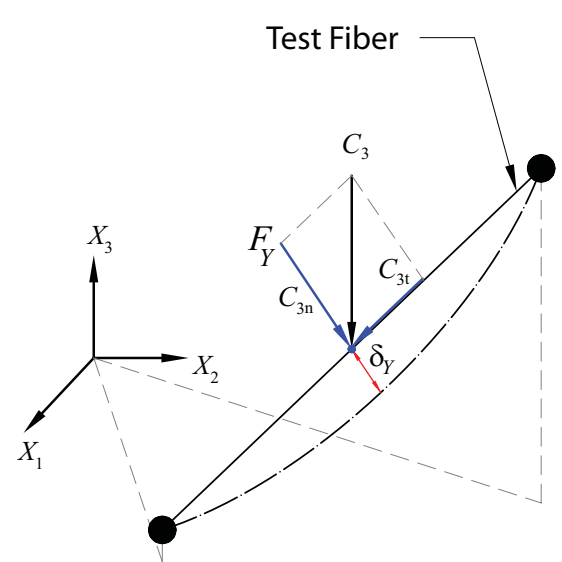

Fig. 3 Deformation of a fiber beam model under contact forces.

If we consider a control volume with unit cross section area confined between two planes, both normal to $X_{3}$, at a distance equal to the vertical projection of a random fiber, the pressure flowed into the network transfers to the contact forces between all interacting elements in the volume. Mathematically, the pressure $P$ can be expressed as

$$
d P=n_{\bar{\lambda}_{3}} d C_{3}=N_{v} V_{\bar{\lambda}_{3}} d C_{3} \quad \text { where } \quad V_{\bar{\lambda}_{3}}=\bar{\lambda} K_{3}
$$

in which $n_{\bar{\lambda}_{3}}$ is the number of contact points in the volume $V_{\bar{\lambda}_{3}}$ with unit cross section area and the height $\bar{\lambda}_{3}$ equal to the mean projection of the random fiber length on third direction, $K_{3}$ is a structural parameter that averages the projection of mean fiber length in the third direction, and $C_{3}$ is the contact force between interacting fibers in the third direction. It should be noticed that there is a relation between $N_{v}$ and $\bar{\lambda}$ in terms of structural features of the network.

Finally, the connection between bulk deformation and average contact force in the third direction, assuming an affine deformation rule, can be acquired using Eq. (4) for the fiber segment shown in Fig. 3:

$$
\delta_{3}=\frac{C_{3} \bar{\lambda}^{3}}{24 E I} M_{3}, \quad M_{3}=\int_{0}^{\pi} \int_{0}^{\pi} \sin ^{2} \theta \Omega(\phi, \theta) \sin (\theta) \mathrm{d} \theta \mathrm{d} \phi
$$

where $\delta_{3}$ is the macroscopic deformation in the third direction.

Using the idea of a binomial distribution of the number of fiber contacts mentioned in Section 2.1 and the blocked fiber length (the length of fiber that has been already blocked by present contacts and is not available for a new contact to be formed), along with the theoretical differential relation between macroscopic pressure and bulk deformation mentioned in Eq. (5), Neckář et al. [11] developed a modified pressure-volume fraction relation as follows:

$$
P=K_{p}\left(\frac{\eta}{Q \gamma(1-\eta)}\right)^{3}\left\{(1-\eta)\left[2+(3 / 2) \frac{\eta}{1-\eta}\right]-\frac{\eta}{Q \gamma v_{f}}\right\}, \eta=1-e^{-Q \gamma v_{f}}
$$

in which $K_{p}$ is an uncertainty parameter, representing fiber modulus of elasticity, that accounts for all assumptions, approximations, and averaging errors that appear in the mechanistic model, and $Q=2$ for spatial random fiber networks [11]. 
To the knowledge of the authors, Neckár et al. [11] is the only model that is able to represent the compression behavior of felt, by which realistic optimized parameters can be obtained in the process of the parameter identification problem [3].

\subsection{Nonlinear hysteretic modeling}

Hysteresis is a recurring phenomenon in the compressional behavior of fiber assemblies, which has been confirmed by many experiments done on different types of fiber networks $[5,12]$. The source of hysteresis can be friction between fibers sliding during network deformation, or structural damping in fiber deformation. Sliding between fibers is likely to happen, particularly in the compression of fiber assemblies at large deformations. It is crucial to note that due to friction, the evolution of network structure in loading and unloading stages would be fairly different, which indirectly contributes to generating hysteresis. In this section, a

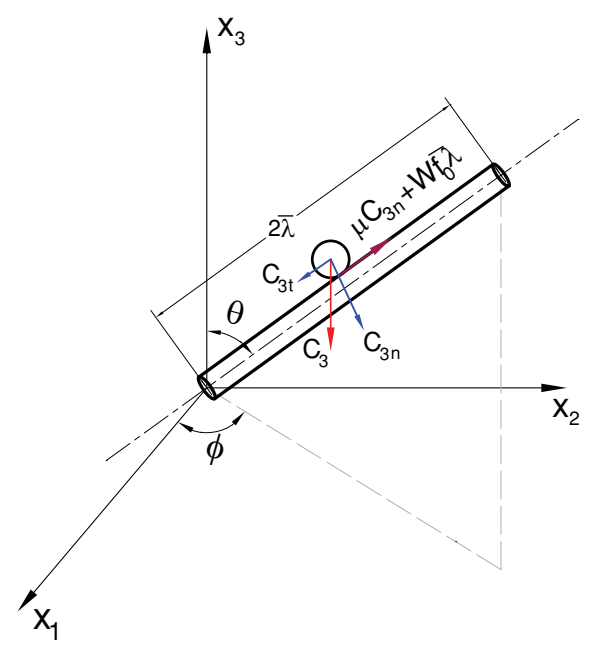

Fig. 4 Contact forces developed in fiber contacts.

nonlinear hysteresis model for the compression of a fiber network, derived by Carnaby et al. [13], is presented. A novel technique, which is a creative combination of the mechanistic model presented by Neckář and the hysteretic approach proposed by Carnaby, is introduced to estimate the compression behavior of felt during the simulation of multibody systems with felt-lined interacting bodies.

To develop a mechanistic model of felt compression including nonlinear hysteresis, some key assumptions should be considered [13], referring to the schematic of frictional fiber interaction at a typical contact point as depicted in Fig. 4:

1. There exist two types of fiber contacts: sliding and non-sliding. Depending on the orientation configuration of the fibers and the magnitude of normal force between interacting fibers, a criteria for fiber sliding can be established.

2. A subnetwork of fibers with non-sliding contacts absorbs a portion of the external work done on the bulk network as fiber bending elastic energy. 
3. Sliding contacts account for the remaining portion of the energy flow into the fiber mass in an irreversible form.

4. There is some withdrawal force on fibers, even in the absence of any external load applied to the fiber mass, due to mutual interference and friction between interacting fibers.

5. The fibers slide when the tangential force exceeds the friction force and withdrawal force between interacting fibers.

According to Coulomb's dry friction rule, the contacting fiber in Fig. 4 starts to slide on the test fiber if the tangential force exceeds the microscopic resisting force between the interacting surfaces that are in atomically close contact. That is [13], when:

$$
C_{3 t} \geq \mu C_{3 n}+W f_{0} \bar{\lambda} \Rightarrow C_{3} \cos (\theta) \geq \mu C_{3} \sin (\theta)+W f_{0} \bar{\lambda}
$$

where $C_{3 t}$ is the tangential force on the test fiber, $C_{3 n}$ is the normal force, $C_{3}$ is the contact force in the third direction, $\mu$ is the coefficient of friction, and $W f_{0}$ is the withdrawal force per unit length of fiber in the absence of external loads on the fiber mass. According to Eq. (8), the critical angle $\theta_{c r}$ at which sliding occurs can be calculated, so the fibers with polar angle less than $\theta_{c r}$, which is a function of mean contact force $C_{3}$, slide. There is no slippage for the fibers with $\theta \geq \theta_{c r}$.

The main source of withdrawal force comes from the fact that due to fiber interaction and friction, the fibers still have some residual bending energy in the absence of external loads on the fiber network. In fact, interactions between fibers prevent them from reaching their lowest energy level. There are some experiments done by Grosberg and Smith [14] to estimate fiber withdrawal force. Theoretically, they proved that the withdrawal force $W f$ per unit length of fiber is approximately proportional to the external pressure $P ; W f_{0}$ is its finite value at $P=0$.

In general, the macroscopic external load on the network must flow through fiber contact points. If $r_{s c}$ is the proportion of contact points that slide, we can rewrite Eq. (5) in case of loading as

$$
P_{\text {loading }}=\left(1-r_{s c}\right) n_{\bar{\lambda}_{3}} C_{n s}+r_{s c} n_{\bar{\lambda}_{3}} C_{s} \Rightarrow P_{\text {loading }}=\sigma_{e l}+r_{s c} n_{\bar{\lambda}_{3}} C_{s}
$$

in which $C_{s}$ is the average contact force per sliding contact points, $C_{n s}$ is the average contact force per non-sliding ones, and $\sigma_{e l}$ is the elastic stress developed in the network during loading.

For the unloading stage, we have

$$
P_{\text {unloading }}=\left(1-r_{s c}\right) n_{\bar{\lambda}_{3}} C_{n s}-r_{s c} n_{\bar{\lambda}_{3}} C_{s} \Rightarrow P_{\text {unloading }}=\sigma_{e u}-r_{s c} n_{\bar{\lambda}_{3}} C_{s}
$$

in which $\sigma_{e u}$ is the elastic stress developed in the network during unloading. It is obvious that parameters in Eqs. (9) and (10) are different in the cases of loading and unloading, i.e. $\sigma_{e l} \neq \sigma_{e u}$, and the effect of friction is not the same during loading and unloading.

Estimating $\theta_{c r}$ using Eq. (8), it is easy to find the proportion of fibers that slide:

$$
r_{s c}=\int_{0}^{\theta_{c r}} \int_{0}^{\pi} \Omega(\phi, \theta) \sin (\theta) \mathrm{d} \theta \mathrm{d} \phi
$$


Contacting fibers slide when motion between the interacting fibers is impending; at this point, the magnitude of contact force approaches its threshold value, also called the limiting friction:

$$
C_{s} \cos \left(\theta_{c r}\right)=\mu C_{s} \sin \left(\theta_{c r}\right)+W f_{0} \bar{\lambda}
$$

Averaging all resistances per sliding contact point over the orientation space results in:

$$
C_{s}=\int_{0}^{\theta_{c r}} \int_{0}^{\pi} \frac{W f_{0} \bar{\lambda}}{\cos (\theta)-\mu \sin (\theta)} \Omega(\phi, \theta) \sin (\theta) \mathrm{d} \theta \mathrm{d} \phi
$$

Furthermore, the corresponding mean non-sliding contact force can be evaluated as

$$
C_{n s}=\frac{\left(P \pm r_{s c} n_{\bar{\lambda}_{3}} C_{s}\right)}{\left(1-r_{s c}\right) n_{\bar{\lambda}_{3}}}
$$

in which $P$ can be either $P_{\text {loading }}$ or $P_{\text {unloading }}$, depending on the sign of macroscopic load rate that signifies the state of loading.

During unloading, the bending energy stored in the test fiber causes the contacting fiber to recover; it applies a normal force to the slipping fiber. Even in the absence of normal force, the contacting fiber does not move back up to the test fiber. So, it is fairly reasonable to use the same logic that Carnaby and Pan [13] assumed in the recovery stage, namely that the sliding force $C_{s}$ carries the resistance $W f_{0} \bar{\lambda}$ at the interfaces. In other words,

$$
C_{s} \cos \left(\theta_{c r}\right)=W f_{0} \bar{\lambda}
$$

This is in agreement with the withdrawal experiments, showing that in the absence of macroscopic load on the fiber mass, there exists some finite contact force at contact points. Consequently, the resistance force per slipping contact during unloading, averaged over the orientation space will be:

$$
C_{s}=\int_{0}^{\theta_{c r}} \int_{0}^{\pi} W f_{0} \bar{\lambda} \sec (\theta) \Omega(\phi, \theta) \sin (\theta) \mathrm{d} \theta \mathrm{d} \phi
$$

\section{Integration of micromechanical contact model with multibody dynamic simulation}

The micromechanical model presented in Section 2.2, based on a binomial distribution of the number of fiber contacts, combined with the compression hysteresis model built on fiber-to-fiber friction and structural parameter difference in loading-unloading stages, can be a promising approach in mechanistic modeling of multibody systems with interactions between bodies lined with fiber networks. We developed an algorithm to incorporate the hysteresis phenomenon into the micromechanical model using the experimental data for felt, so that the generated model can be utilized in multibody dynamic simulations. Experimental data extracted by Stamm [5], for compression tests on wool felt samples with different volume fractions, have been used as reference data in this research. As demonstrated in Fig. 5, this is a two-step process, as explained next. 
3.1 Micromechanical parameter estimation

For every piece of felt, experimental loading-unloading curves are used to: (i) extract the elastic portions of compression hysteresis, which are used to identify the parameters of the mechanistic pressure-volume fraction relation presented in Eq. (7), and (ii) estimate the critical angle of sliding in terms of volume fraction, which is utilized to evaluate the inelastic portions of hysteresis (due to friction between sliding fibers) in which the energy is not conserved.

To this end, a load increment of the mean value of contact force $\Delta C_{3}$ is considered, for which the critical angle of sliding $\theta_{c r}$ and the proportion of contact points that slide $r_{s c}$ are calculated using Eqs. (12) and (11), respectively. Accordingly, the loading pressure $P_{\text {loading }}$ and the corresponding elastic portion $\sigma_{e l}$ can be calculated using Eq. (9). We then use the experimental data to specify the deformation (volume fraction) corresponding to the obtained loading pressure, elastic portion, and critical angle of sliding. The same process is followed for the recovery stage in evaluating $P_{\text {unloading }}$ and $\sigma_{e u}$, employing Eq. (10). Continuing the scenario of increasing the mean value of contact force, the elastic portion of the bulk pressure, $\sigma_{e l}$ and $\sigma_{e u}$, along with the critical angle of fiber sliding, $\theta_{c r}$, are calculated in terms of volume fraction for loading and unloading stages. These estimated parameters will be used in the multibody simulations.

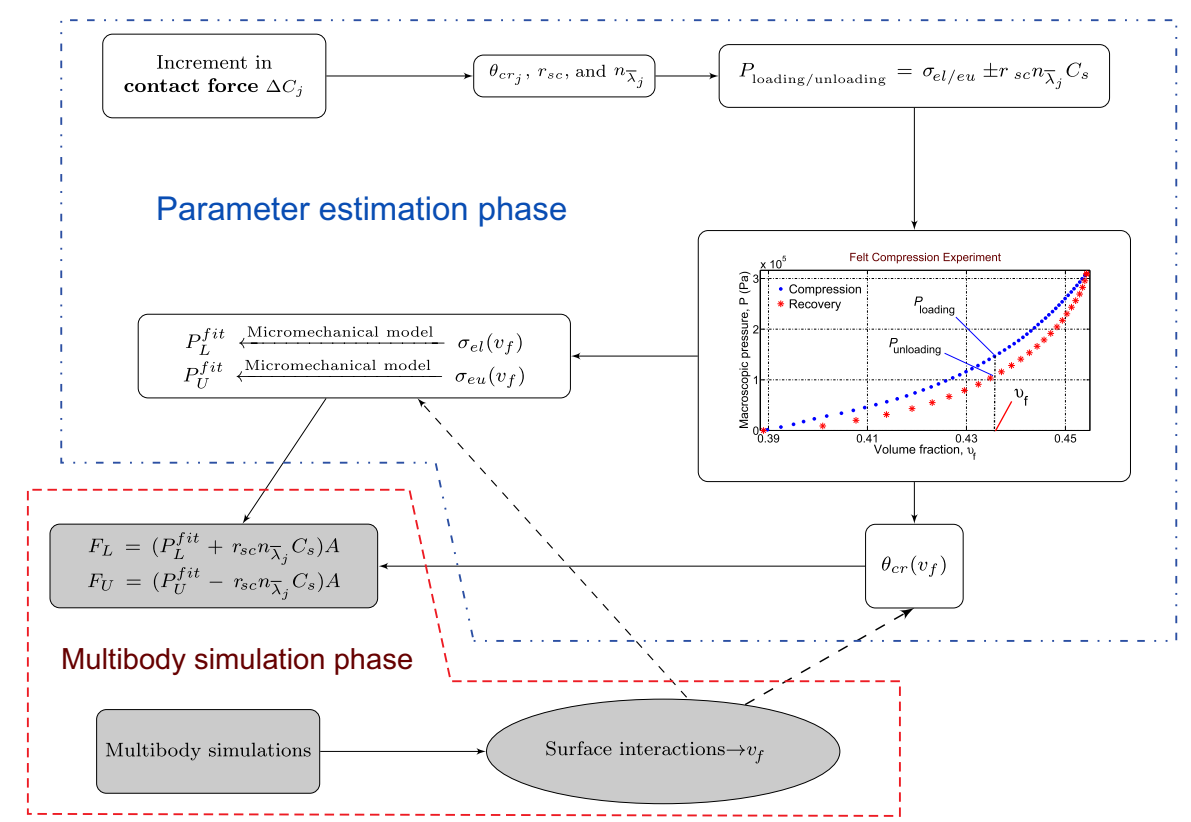

Fig. 5 The procedure developed to apply the proposed micromechanical model in multibody dynamic simulations.

It should be noted that the network is treated as a bonded nonwoven assembly, considering the elastic portion of macroscopic loading-unloading pressure. Hence, 
the micromechanical model based on a binomial distribution of number of fiber contacts, represented by Eq. (7), can be used to identify $K_{p}$ and $\gamma$ for the obtained elastic pressure data. Different optimized parameters signify a different structural behavior of the network in compression-recovery stages. In short, this mechanistic model is based on bending of the network constituent fibers at the microscale. The critical angle of sliding, $\theta_{c r}$, is stored as a structure parameter in terms of volume fraction to avoid iterations at each time step of multibody dynamic simulations.

The procedure has been demonstrated in Fig. 5; the white blocks of the flowchart (white blocks) show how the elastic portion of loading and unloading curves is calculated for a specific range of deformation, and then the parameters of the micromechanical model (Eq. (7)) are estimated. The parameters are estimated by minimizing the objective function representing root square error between the physics-based curve (Eq. (7)) and experimental data for each felt sample in contact interfaces of the piano action. The minimization algorithm used in this work is Matlab's "fminsearch". The critical angle of sliding is evaluated at each volume fraction and attributed uniquely to the contact interface as a structural parameter for use in the next step.

\subsection{Multibody dynamic simulation}

In multibody simulations, the penetration between interacting bodies or change in volume fraction is calculated at each time step. So, the elastic portion of bulk pressure can be evaluated by the micromechanical model with the parameters identified for different felt interfaces of the multibody system. Expressing the critical angle $\theta_{c r}$ of sliding as a function of volume fraction from our parameter estimation, the effect of friction and contribution of sliding points in the loading-unloading bulk pressure will be included by adding $r_{s c} n_{\bar{\lambda}_{3}} C_{s}$ to the obtained elastic portion $\sigma_{e l}$ in case of loading, and subtracting $r_{s c} n_{\bar{\lambda}_{3}} C_{s}$ from the elastic portion $\sigma_{e u}$ in case of unloading, referring to Eqs. (9) and (10).

Referring to Fig. 5, the obtained results in the previous section 3.1 are utilized in multibody dynamic simulations (grey blocks) to achieve the overall macroscopic loading $\left(P_{L}\right)$ and unloading pressures $\left(P_{U}\right)$, including the elastic portions $\left(P_{L}^{f i t}\right.$ and $\left.P_{U}^{f i t}\right)$ and the fiber-to-fiber friction contribution $\left(+r_{s c} n_{\bar{\lambda}_{3}} C_{s}\right.$ and $\left.-r_{s c} n_{\bar{\lambda}_{3}} C_{s}\right)$, when deformation or volume fraction is known.

Simulation results obtained by minimizing the error between Eq. (7) and the elastic portion of the experimental data, extracted using the above-mentioned procedure, for two felt samples used in piano action mechanisms, along with the identified parameters are shown in Figs. 6 and 7.

\section{Multibody dynamic simulation of a vertical piano action}

To examine the performance of the micromechanical approach in a practical multibody problem, a piano action mechanism in which there are several interacting bodies, covered with felt, is described in this section. The piano action is a complex multibody system consisting of rigid and flexible bodies, revolute and prismatic joints, and different contact interfaces. The process of developing a high-fidelity 


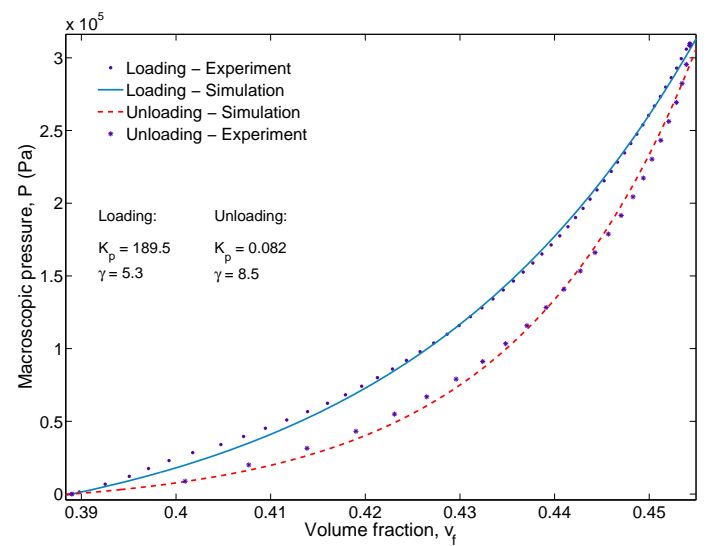

Fig. 6 Loading and unloading experimental data for piano hammer felt with $v_{f 0}=0.389$, along with the identified parameters of the micromechanical model.

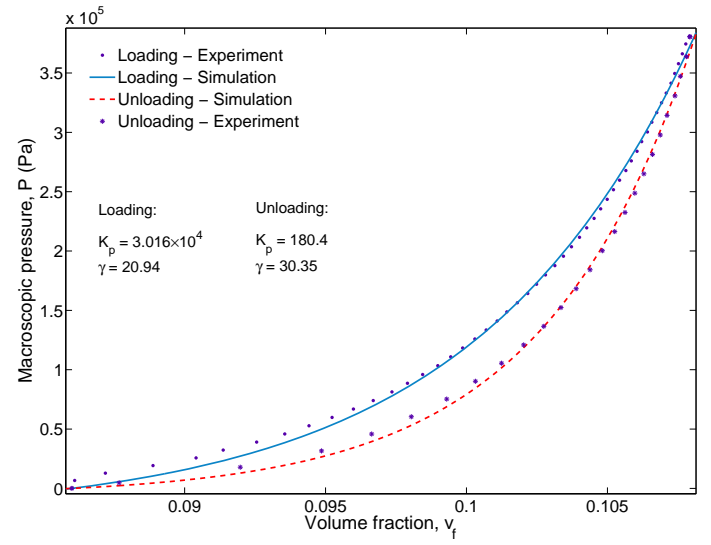

Fig. 7 Loading and unloading experimental data for moderate red felt with $v_{f 0}=0.086$, along with the identified parameters of the micromechanical model.

multibody model of an upright piano action, along with its experimental procedure and validation, are illustrated in the following sections.

4.1 Multibody dynamic model of the piano action

A typical piano action mechanism is shown in Fig. 8, consisting of five main bodies: key, whippen, jack, hammer, and damper. There are two other components, butt spring and bridle strap, in the action that control the hammer motion. A simple model of a vertical piano action mechanism was developed by Masoudi et al. [15], in which all the main bodies were considered to be rigid and the string was replaced with a rigid stop. The model fidelity was increased in other works by the authors [3, 16], considering hammer-string interaction, hammer shank flexibility, backcheck wire flexibility, and a sophisticated key pivot model. They thoroughly studied the effect of bridle strap and butt spring on the hammer motion. Moreover, an 


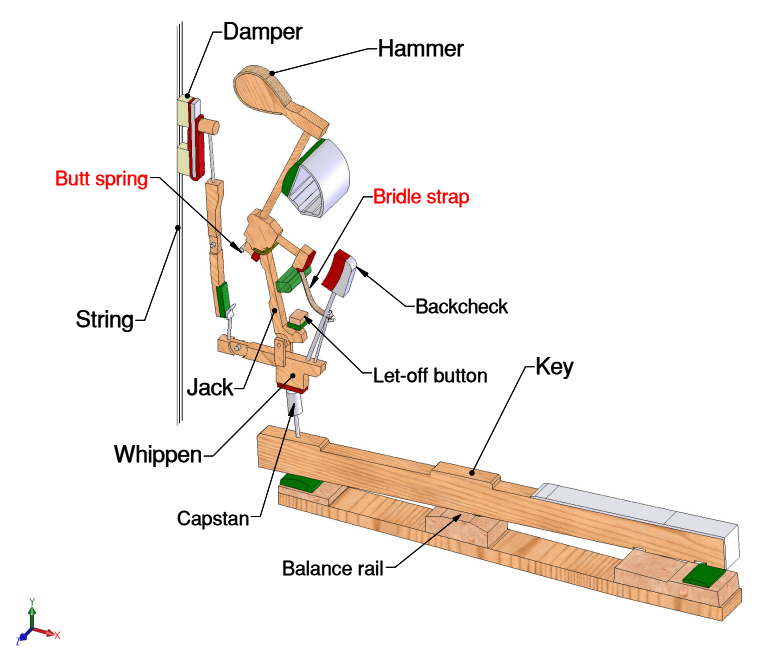

Fig. 8 Components and configuration of a typical vertical piano action mechanism (Essex EUP-123).

empirical model in which an exponential curve was fitted to experimental loading and unloading data for every pair of contact points, was utilized to include contact dynamics in the model. The lack of physical significance for contact modeling was evident in Ref. [16], which is rectified in the current paper.

MapleSim/Multibody [17], a software based on the graph-theoretic approach, is used to automatically generate symbolic equations governing the dynamic behavior of the system. The equations of motion are formulated as an optimized set of twelve ordinary differential equations (ODEs), which in state-space form can be written as

$$
\dot{Q}=\boldsymbol{A}_{M} Q+\boldsymbol{F}_{M}
$$

in which

$$
\boldsymbol{A}_{M}=\left[\begin{array}{ll}
O_{12 \times 12} & \boldsymbol{I}_{12 \times 12} \\
O_{12 \times 12} & 0_{12 \times 12}
\end{array}\right] \quad \text { and } \quad \boldsymbol{F}_{M}=\left\{\begin{array}{c}
0_{12 \times 1} \\
\boldsymbol{M}^{-1} \boldsymbol{F}
\end{array}\right\}
$$

$\boldsymbol{Q}=\left\{\boldsymbol{q}^{\mathrm{T}} \dot{\boldsymbol{q}}^{\mathrm{T}}\right\}^{\mathrm{T}}$ is the state vector of generalized coordinates and speeds, $\boldsymbol{I}$ is the identity matrix, $\boldsymbol{M}$ is the mass matrix, $\boldsymbol{F}=\boldsymbol{F}(\boldsymbol{q}, \dot{\boldsymbol{q}}, t)$ is the vector of generalized forces containing quadratic velocity terms, gravitational terms, contact forces, and friction torques, and $\boldsymbol{q}$ is the generalized coordinate vector consisting of five terms for the rigid-body motions of the five bodies, as well as vibrations of the flexible components, i.e. $u_{w}$ and $u_{s}$ (the longitudinal generalized elastic coordinates) and $v_{w_{i}}$ and $v_{s_{i}}, i=1,2$ (lateral elastic coordinates) for the flexible backcheck wire and flexible hammer shank, respectively.

A schematic of the piano action mechanism, along with the tracking points used in the experimental measurement of position and speed of the action components, is shown in Fig. 9. The direction of rotation of various components of the action in response to an applied input force on the key, with a load sensor attached to the key, is also illustrated in the schematic figure. 


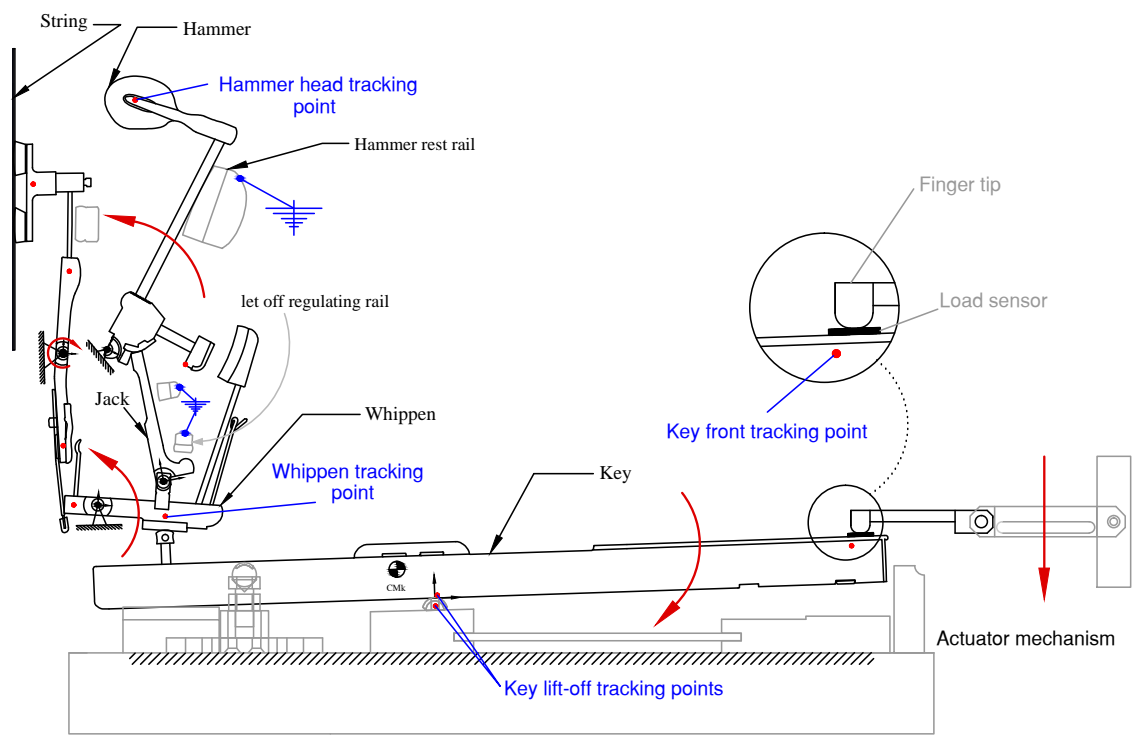

Fig. 9 A Schematic of the piano action mechanism, illustrating the location of tracking points on the components of the action, along with the direction of rotation of the components (see arrows) when a force is applied on the key front by the actuator mechanism.

\subsection{Hammer-string interaction}

Hammer-string interaction was included in the model by adapting the technique of Vyasarayani et al. [18] to the vertical action. Dynamic equations governing the lateral displacement of the string, $x(y, t)=\phi^{\mathrm{T}}(y) \eta(t)$, subject to the timedependent hammer-string normal contact force, $f_{n}(t)$, can be written in statespace form:

$$
\dot{\boldsymbol{\Psi}}=\boldsymbol{A}_{S} \boldsymbol{\Psi}+\boldsymbol{F}_{S}
$$

in which $y$ is the distance from one end of the string along its axis in the initial (undeformed) state, $\phi$ is the vector for undamped mass normalized mode shapes for the string, $\eta$ is the vector for string vibration modes, $\boldsymbol{\Psi}=\left\{\eta^{\mathrm{T}} \dot{\eta}^{\mathrm{T}}\right\}^{\mathrm{T}}$,

$$
\boldsymbol{A}_{S}=\left[\begin{array}{cc}
0_{N \times N} & \boldsymbol{I}_{N \times N} \\
-\boldsymbol{R}_{N \times N} & -\boldsymbol{P}_{N \times N}
\end{array}\right], \quad \text { and } \quad \boldsymbol{F}_{S}=\left\{\begin{array}{c}
0_{N \times 1} \\
\boldsymbol{F}_{\mathrm{norm}}
\end{array}\right\}
$$

where $\boldsymbol{R}=\operatorname{diag}\left(\omega_{1}^{2}, \ldots, \omega_{N}^{2}\right), \boldsymbol{P}=\operatorname{diag}\left(2 \xi_{1} \omega_{1}, \ldots, 2 \xi_{N} \omega_{N}\right)$, and

$$
\boldsymbol{F}_{\text {norm }}=\left\{\phi_{1}\left(y_{c}\right) f_{n}(t), \ldots, \phi_{N}\left(y_{c}\right) f_{n}(t)\right\}^{\mathrm{T}}
$$

where $\xi_{j}$ is the modal damping, $\omega_{j}$ is the natural frequencies, $y_{c}$ is the hammerstring contact location along undeformed string axis with respect to the string reference frame, and $N$ is the number of modes. The closed-form expressions for 
natural frequencies and mass-normalized mode shapes given by Fletcher [19] are as follows:

$$
\omega_{j}=\frac{\pi j}{L} \sqrt{\frac{T}{\mu}\left(1+\varepsilon \pi^{2} j^{2}\right)}, \quad \text { and } \quad \phi_{j}(y)=\sqrt{\frac{2}{\mu L}} \sin \left(j \pi \frac{y}{L}\right)
$$

In Eq. (21), $\mu$ is the linear density, $L$ is the string speaking length, $T$ is the tension in the string, $\varepsilon=\pi d^{4} E / 64 L^{2} T$ is called the inharmonicity index, $E$ is Young's modulus of elasticity, and $I$ is the second moment of area of cross section.

\subsection{Mechanistic contact dynamics}

There are 16 contact locations in the action, lined with felt or leather, with interfaces of different geometries. Two major steps should be followed to predict the dynamics of contact between interacting bodies in the piano action: contact detection and contact force calculation. A fully geometrical technique is used to detect contacts, or the extent of penetration between colliding bodies. This calculation obviously depends only on the geometry, location, and (in some cases) the curvature of the contact interfaces. Details of the contact locations and geometries can be found in Refs. $[3,16]$. To estimate contact forces, the proposed hysteretic micromechanical model discussed in Sec. 3 is applied to the action, and its performance in multibody system simulations will be analyzed. To include partial loading and unloading during contacts, a mathematical relation is utilized $[3,16]$ :

$$
F_{n}\left(\varepsilon_{p}, \nu_{n}\right)= \begin{cases}F_{L}\left(\varepsilon_{p}\right), & \text { if } \nu_{n} \geq 0 \\ F_{L}\left(\varepsilon_{p}\right)+\delta_{L U}\left(\varepsilon_{p}\right) \tanh \left(\alpha \nu_{n}\right), & \text { if } \nu_{n}<0\end{cases}
$$

where $F_{n}$ is the normal contact force, $\varepsilon_{p}$ is the penetration depth, $\delta_{L U}\left(\varepsilon_{p}\right)=$ $F_{L}\left(\varepsilon_{p}\right)-F_{U}\left(\varepsilon_{p}\right)$ is the difference between the loading and unloading curves at each penetration depth, $\alpha$ is a constant that controls the transition speed between loading and unloading states, and $\nu_{n}$ is the normal speed between the two surfaces at the contact point, which also influences the transition. The relation defined in Eq. ( 22) resembles the plot in Fig. 2, which confines the partial loading and unloading curves to lie within the experimental hysteresis loop used to obtain loading and unloading curves-i.e., $F_{U}\left(\varepsilon_{p}\right) \leq F_{n}\left(\varepsilon_{p}\right) \leq F_{L}\left(\varepsilon_{p}\right)$.

\subsection{Experimental procedure - parameter estimation}

A prototype of the piano action was assembled for a single-key mechanism, along with a prescribed vertical motion applied to the mechanism by a linear motor. A precise description of the experimental procedure has been presented in another work by the authors [20]. Displacements of different bodies were measured by tracking marking points (shown in Fig. 9), made of minute paper circles glued to the action components at desired locations, using a Photron Fastcam PCI1280 high-speed camera. Input force to the action was estimated using a force sensor mounted on the actuator, reproducing the force applied by the pianist's 
finger. The obtained force sensor data and position data from different cameras were synchronized. All the parameters for the components, including mass and geometrical properties, flexible components and string parameters, and contact points parameters were measured experimentally [20].

\subsection{Simulation results and experimental validation}

Applying a force on the key front, the key rotates clockwise (as shown in Fig. 9), hits the felt underneath, bounces, then rests on the front rail punching. Simultaneously, the hammer rotates counterclockwise, flies into the string, rebounds, and gets captured by the backcheck. The whippen transmits the motion generated by the key to the hammer, so an accurate interaction model between the key and whippen (as well as other contact locations) has a pivotal role in the precise response of the system.

The simulation stages for multibody dynamics of the piano action is shown in Fig. 10, which is a more detailed flowchart representing the computational scheme used in multibody simulation phase of Fig. 5 (grey blocks). In each step of sim-

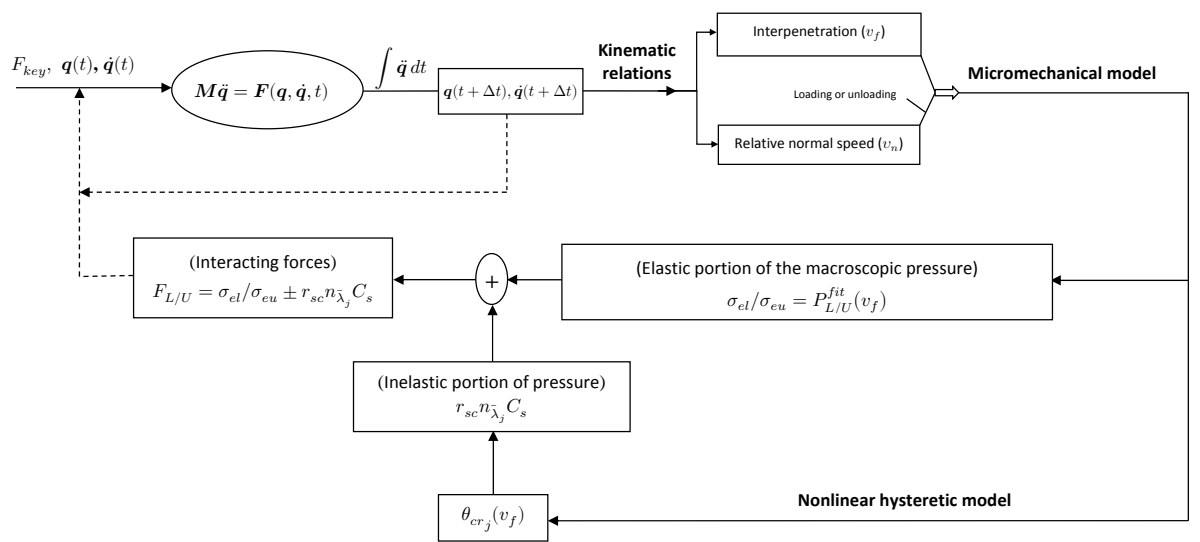

Fig. 10 The procedure for dynamic simulation of the vertical piano action incorporating the micromechanical contact model at interfaces.

ulation, the generalized coordinates and speeds are computed using a numerical integration method. By applying kinematic relations, along with these coordinates and speeds, the penetration depth and relative normal speed between interacting bodies at the point of contact will be estimated. The normal speed distinguishes between loading and unloading stages. On one hand, penetration depth, or volume fraction, is used to calculate the elastic portion of the pressure due to the interpenetration between bodies (Eq. (7) with the identified parameters for the felt-lined interfaces), and on the other hand, to obtain critical angle of sliding, which is used to estimate the contribution of friction in the overall pressure generated between contacting bodies. The obtained overall contact pressure/force between the bodies (calculated by adding the hysteresis part to the elastic part), along with the 

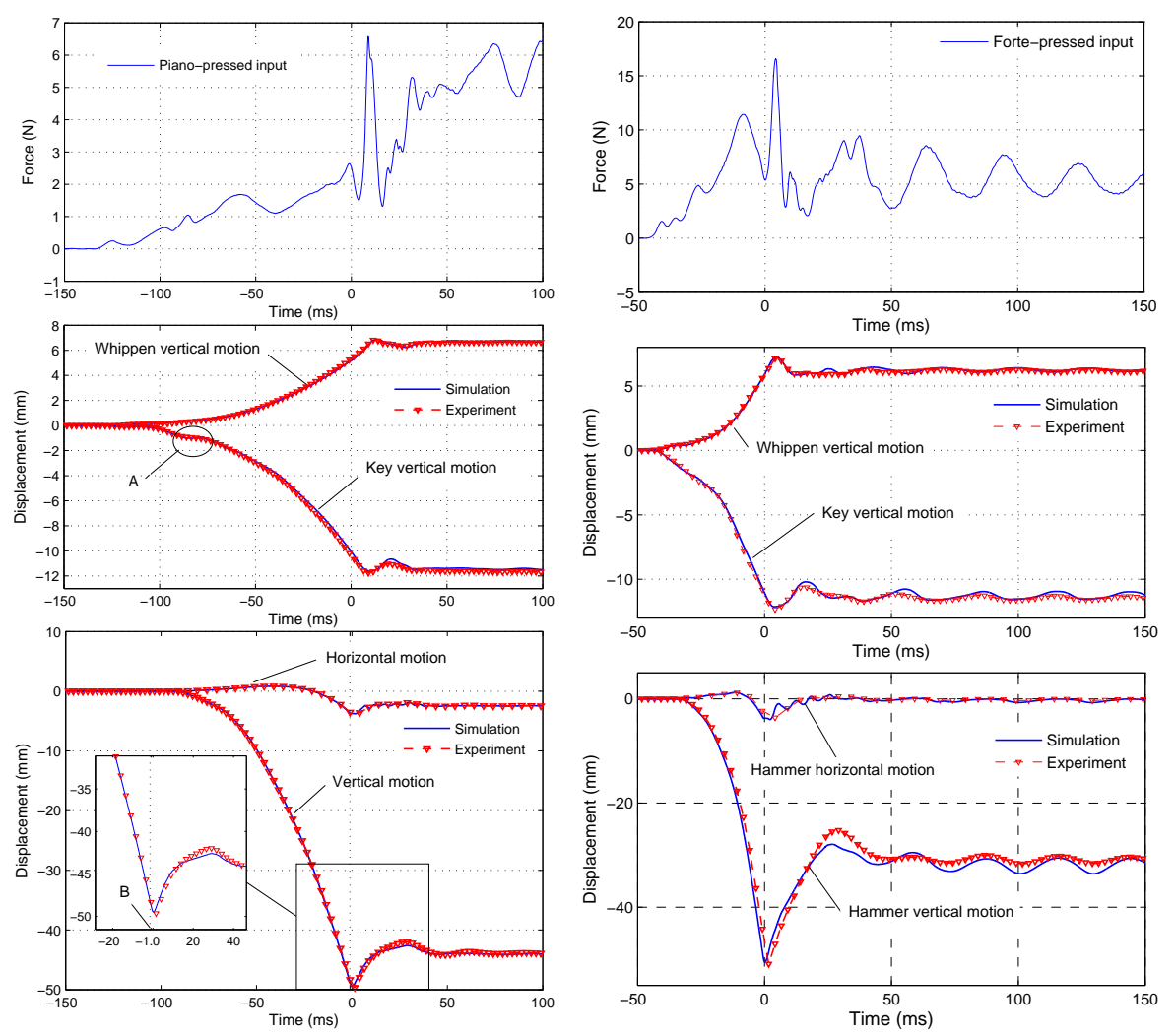

Fig. 11 Piano-pressed input force profile (top) and simulated and experimental vertical displacement of key front and whippen (middle) and hammer tip (bottom) tracking points. Time zero corresponds to the moment of maximum hammer-string contact force.

Fig. 12 Forte-pressed input force profile (top) and simulated and experimental vertical displacement of key front and whippen (middle) and hammer tip (bottom) tracking points. Time zero corresponds to the moment of maximum hammer-string contact force.

generalized coordinates and speeds is fed-back to the input for the next step of simulation.

Two different inputs introduced by Masoudi et al. $[3,20]$ are utilized to simulate the vertical action, incorporating the micromechanical contact dynamic model developed in previous sections for the hammer-string interaction. The input profiles have been derived from force sensor data attached to the key front, which imitate the pianist finger force when playing a note with different intensities, i.e. piano-pressed (soft) and forte-pressed (strong). Figures 11 and 12 show the time history of the piano-pressed (left) and forte-pressed (right) inputs, along with the corresponding key, whippen, and hammer motions for the simulated model and experimental results. Since the micromechanical contact model represents the real hysteresis curves corresponding to hammer-string contact point, the results are very similar to the ones presented in Ref. [20] which is based on a math-based contact model, i.e. curve-fitting to the experimental felt compression data. The errors between the two models, i.e. the math-based model presented in Ref. [20] 
and the physics-based model proposed in this work, are shown in Figs. 13 and 14 for piano and forte inputs, respectively. The small error values between the contact models result in a very similar trajectories for the key, whippen, and hammer, compared to the ones represented in Ref. [20]. The main difference is a longer sim-

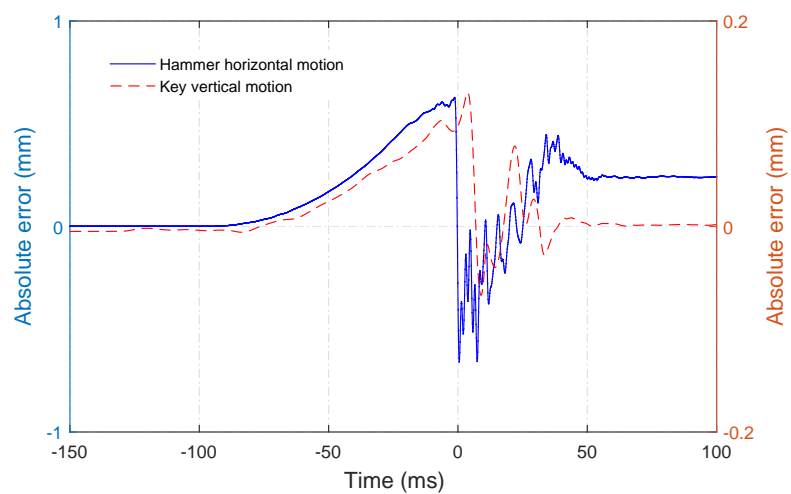

Fig. 13 Absolute error values between the model presented in Ref. [20] and the model proposed in this work for hammer horizontal motion and key vertical motion in case of pianopressed input.

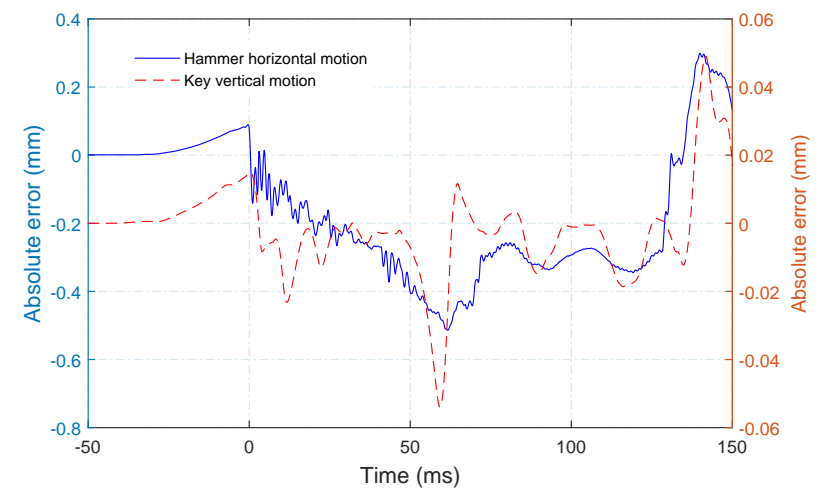

Fig. 14 Absolute error values between the model presented in Ref. [20] and the model proposed in this work for hammer horizontal motion and key vertical motion in case of fortepressed input.

ulation time due to extensive computational effort in estimating force-penetration relations for the physics-based contact model during the simulation. Using a 2.40$\mathrm{GHz}$ quad-core processor, the simulation times for the piano and forte inputs were $1113 \mathrm{~s}$ and $1595 \mathrm{~s}$, respectively. Although these simulations took longer than those using the mathematical contact model, where the CPU running times were, respectively, $262 \mathrm{~s}$ and $269 \mathrm{~s}$ for piano and forte inputs, this technique is still encouraging for multibody simulations. Parallel programming techniques appear to be necessary to acquire the simulation results in a reasonable amount of time. 
It should be noted that point B in Fig. 11 shows the instant of first hammerstring contact. In the same figure, point $\mathrm{A}$ is the time that the jack starts to push the hammer, thereby a considerable change in the speed of the key front can be observed. The simulated trajectories accurately follow the experimental ones, which signifies the efficacy of the modeling schemes (multibody model and contact dynamic model) and accuracy of the measured parameters. The simulation time for the multibody system with the micromechanical contact model is about 5 times slower than the curve-fit used in [20]. From the stand point of multibody dynamics, the acquired simulation time for such a high-fidelity model including a fully mechanistic contact process is quite satisfactory.

\section{Summary and Conclusions}

A micromechanical model for nonlinear compression hysteresis of random fiber networks was developed and examined on felt, a fiber assembly with random structure, exhibiting velocity-independent hysteretic behavior in compressive loading schemes. Applicability of the proposed model in contact dynamics of interacting bodies (covered with such materials) in multibody systems was the main motivation for this work. A binomial distribution of fiber contacts, together with the Coulomb friction criterion for fiber sliding, have been used to construct the nonlinear hysteretic behavior of the network. Different felt samples were utilized to experimentally establish the performance of the micromechanical model for felt compression. A heuristic procedure was introduced to apply the physics-based continuous contact model in multibody dynamic simulations, in which the tractability of simulation time during interactions between colliding bodies is a challenging issue. To confirm the efficacy of the proposed model, a high-fidelity multibody dynamic model of a vertical piano action with contact interfaces lined with felt or leather was studied in this work. A close agreement between simulation and experimental results for significantly different inputs to the action, along with an acceptable simulation time, affirms the accuracy and performance of the proposed micromechanical contact model in multibody system dynamics.

Acknowledgements The authors gratefully acknowledge the financial support of the Natural Sciences and Engineering Research Council of Canada (NSERC).

\section{References}

1. Gilardi, G., Sharf, I.: Literature survey of contact dynamics modelling. Mechanism and Machine Theory. 37(10), 12131239 (2002)

2. Flores, P., Machado, M., Silva, M.T.: On the continuous contact force models for soft materials in multibody dynamics. Multibody System Dynamics. 25(3), 357375 (2011)

3. Masoudi, R.: Micromechanics of Fiber Networks Including Nonlinear Hysteresis and its Application to Multibody Dynamic Modeling of Piano Mechanisms. PhD Thesis, University of Waterloo, Waterloo, Ontario, Canada (2012)

4. Lehmberg, W.H.: Mechanical Properties and Uses of Wool Felt. Technical Report, New York: The Felt Association Inc., 1945.

5. Stamm, W.: Compressional Behavior of Felt. Diploma Thesis, University of Waterloo, Waterloo, ON, Canada, 2004.

6. van Wyk, C.M.: Note on the compressibility of wool. Journal of the Textile Institute, 37(12), T285-T292 (1946) 
7. Neckář, B.: Compression and packing density of fibrous assemblies. Textile Research Journal. $67,123-130(1997)$

8. Komori, T., Itoh, M.: A model analysis of the compressibility of fiber assemblies. Textile Research Journal. 62, 567-574 (1992)

9. Komori, T., Itoh, M.: Analyzing the compressibility of a random fiber mass based on the modified theory of fiber contact. Textile Research Journal. 67, 204-210 (1997)

10. Alkhagen, M., Toll, S.: Micromechanics of a compressed fiber mass. Journal of Applied Mechanics. 74, 723-731 (2007)

11. Neckář, B., Ibrahim, S.: A contribution to van Wyk's theory of contacts between fibers. In: Proceedings of The Fiber Society 2003 Spring Symposium Programme, Advanced Flexible Materials and Structures: engineering with fibers, June 30 - July 2, Loughborough University, UK (2003)

12. Poquillon, D., Viguier, B., Andrieu, E.: Experimental data about mechanical behaviour during compression tests for various matted fibres. Journal of Materials Science. 40(22), 5963-5970 (2005)

13. Carnaby, G.A., Pan, N.: Theory of the compression hysteresis of fibrous assemblies. Textile Research Journal. 59(5), 275-284 (1989)

14. Grosberg, P., Smith, P.A.: The strength of slivers of relatively low twist. Journal of the Textile Institute. 57(1), T15-T23 (1966)

15. Masoudi, R., Birkett, S.H., McPhee, J.: Dynamic model of a vertical piano action mechanism. In: Proceedings of the ASME IDETC/CIE 2009, Aug. 30-Sep. 2, 2009, San Diego, CA, (DETC2009-87680), 389-398 (2009)

16. Masoudi, R., Birkett, S. H., McPhee, J.: Mechanistic multibody model for simulating the dynamics of a vertical piano action. ASME Journal of Computational and Nonlinear Dynamics. 9(3), 031014(10 pages) (2014).

17. Maplesoft: MapleSim User's Manual. Waterloo, Ontario, Canada, http://www.maplesoft.com/products/maplesim/ (2014).

18. Vyasarayani, C.P., Birkett S.H., McPhee, J.: Modelling the dynamics of a compliant piano action mechanism impacting an elastic stiff string. Journal of Acoustical Society of America. 125(6), 4034-4042 (2009)

19. Fletcher, H.: Normal vibration frequencies of a stiff piano string. The Journal of the Acoustical Society of America. 36(1), 203-209 (1964)

20. Masoudi, R., Birkett, S. H.: Experimental validation of a mechanistic multibody model of a vertical piano action. ASME Journal of Computational and Nonlinear Dynamics. accepted for publication (2014) 